\title{
FRACTURE RESISTANCES OF CAD/CAM MONOLITHIC CROWNS WITH DIFFERENT OCCLUSAL THICKNESS
}

\author{
Amira Mohamed Elsharkawy* and Nasser Hussein Ali ${ }^{* *}$
}

\begin{abstract}
The aim of this study was to examine the effect of different occlusal thickness on fracture resistances of different monolithic crowns.

Materials and methods: Forty molar crowns were fabricated from four different types of monolithic ceramic blocks ( $n=10)$ : Group I: Vita Enamic (V.enamic), Group II: IPS E-max CAD (e.max), Group III: Celtra Duo(CD), Group IV: Functional explore (f.explore); by means of CAD/ CAM system with two occlusal thickness (1 and $1.5 \mathrm{~mm}$ ). All crowns were cemented on implant abutments using self adhesive resin cement. Combined thermocycling and mechanical loading was performed under a chewing simulator, fracture resistance was examined by universal testing machine. The fractured specimens were inspected by scanning electron microscopy (SEM).
\end{abstract}

Results: The highest fracture resistance was recorded with f.explore group followed by $C D$ group then e.max group while the lowest fracture resistance mean value recorded with V.enamic with statistical significance $(\mathrm{P}=0.0001<0.05)$ for both occlusal thicknesses. The $1.5 \mathrm{~mm}$ occlusal thickness recorded statistically non-significant $(\mathrm{P}=0.2267>0.05)$ higher fracture resistance mean value than $1 \mathrm{~mm}$ occlusal thickness except for f.explore group.

Conclusions: The fracture resistance of monolithic crowns was differently affected by the ceramic materials and changes in occlusal thickness. Within the limitations of this study, all the tested crowns resisted the physiological range of masticatory forces at $1.5 \mathrm{~mm}$ and reduced $(1.0$ $\mathrm{mm}$ ) occlusal thickness.

KEY WORDS: Fracture resistances, monolithic crowns, occlusal thickness.

\section{INTRODUCTION}

Implant-supported prostheses are usually made using computer-aided design/computer-assisted manufacturing (CAD/CAM) technologies. ${ }^{1}$ This $\mathrm{CAD} / \mathrm{CAM}$ process assume to guarantee acceptable marginal gap and acceptable strength due to the absence of air bubbles inside the material. ${ }^{2}$ Accordingly, the fracture resistance of restorations made from CAD/CAM blocks is high compared to those hand-processed by a technician. ${ }^{3}$ The higher

\footnotetext{
* Associate Professor, Fixed Prosthodontics, Faculty of Dentistry, $6^{\text {th }}$ of October University, Giza, Egypt

** Associate Professor, Fixed Prosthodontics, College of Dentistry, MTI University
} 
fracture resistance is of particular importance in the posterior regions of the jaw, where the average masticatory force can be up to 600 Newton. ${ }^{4}$

High survival rates of ceramics used in posterior fixed prosthesis was shown to be comparable to those of conservative single restorations in short-term studies. ${ }^{(5,6)}$ Among the most commonly used ceramic restorations in posterior region are monolithic lithium disilicate and yttria-stabilized zirconia. Both were shown to have satisfactory clinical outcomes. ${ }^{(7)}$

Lithium disilicate glass ceramics have expanded popularity among dentists because of their high mechanical properties, great esthetics potential and wear behavior very similar to opposing dental enamel. ${ }^{\left({ }^{8}\right.}$

LD ceramics can be used with both conventional pressing techniques and Computer Aided DesignComputer Aided Manufacturing (CAD/CAM) fabrication techniques; the latter permit for standardized processing of the material, decrease manufacture time and improve cost effectiveness. ${ }^{(9)}$

A recently developed ceramic called zirconiacontaining lithium silicate (ZLS) relies on the addition of 10 mass percent zirconium oxide to lithium silicate glass compositions. Zirconia acts as nucleating agent but remains in solution in the glassy matrix, with two main consequences: A dual microstructure consisting of very fine lithium metasilicate $\left(\mathrm{Li}_{2} \mathrm{SiO}_{3}\right)$ and lithium disilicate $\left(\mathrm{Li}_{2} \mathrm{Si}_{2} \mathrm{O}_{5}\right)$ crystals is obtained. Ceramic blocks of this material are available for CAD/CAM construction. ${ }^{(10)}$

Recently, a hybrid ceramic with interpenetrating polymer and ceramic networks has been technologically innovative in order to combine the advantages of ceramics which simulate the physical properties of natural teeth and the advantages of polymers that have less brittleness and avoid causing wear on antagonistic tooth. ${ }^{(11)}$ The polymer infiltrated ceramic network (PICN) shows equivalent modulus of elasitcity to human dentin. ${ }^{16,17}$ It has a high hardness due to the limitation of crack propagation caused by the two interconnected phases. ${ }^{(11), 12}$ The energy of external force exerted on the surface can be absorbed by plastic deformation and creep of the PICN. ${ }^{(13)}$

Numerous readings have described that the fracture resistance of posterior ceramics restorations is detrimental to treatment achievement. (14,15) However, there is no agreement on a minimum recommended thickness for monolithic lithium disilicate single crowns that is confirmed by scientific data yet and there is no agreement on how thin restorations can be made ${ }^{(14,16)}$. To date, few laboratory data about the mechanical predictability of monolithic crowns are available in the literature, particularly for the so called "ultrathin" configuration (i.e. up to a thickness of $0.5 \mathrm{~mm}$ ), as well as the validation of their clinical performances in the oral environment ${ }^{(8,9)}$

However, limitation in interocclusal space may dictate a compromise in abutment height as well as occlusal thickness of the final restoration. A materials that show sufficient fracture strength in reduced thickness, could be desirable in various clinical situations. For evaluation of new materials, in vitro tests is required combining reproducible laboratory conditions with basic requirements (occlusal loading, thermocycling) of the clinical situation. ${ }^{(17)}$ The purpose of this study was to investigate the fatigue and fracture resistances of different monolithic crowns fabricated by computeraided design and computer-aided manufacturing (CAD/CAM) with different occlusal thickness. The null hypothesis of the present study was: the fracture loads of the CAD/CAM-fabricated monolithic crowns will not differ by ceramic type and occlusal thickness.

\section{MATERIALS AND METHODS}

Forty implant lab analog (Neobiotech C0., Ltd. Guro-gu, Seoul, Korea) ${ }^{1}$ were attached to implant impression copings (IS Hexed Pick-up Impression coping 4.0/Long) and the implant 
analogs were mounted vertically in resin blocks (Cold cure denture base material, Acrostone Dental Factory) (Fig.:1). After setting of the resin mold, the impression copings were removed and straight IS Cemented Abutment $\left(4.5^{*} 1.0 \mathrm{~mm}, \mathrm{~L}: 7.0 \mathrm{~mm}\right.$ Hex) (Neobiotech C0., Ltd. Guro-gu, Seoul, Korea) were screw attached according to manufacturer's instructions. The abutments were shortened to 4 mm using diamond disc mounted on a low-speed hand piece held by a parallelometr. The screw holes of the abutments were filled with visible light activated composite (Filtek Z350 XT Universal Restorative System (3M-ESPE AG Dental products ST. Paul, MN 55144 USA), (Fig.2) light-cured for $40 \mathrm{~s}$ with a LED curing unit (Elipar S10, 3M ESPE, Seefeld, Germany).

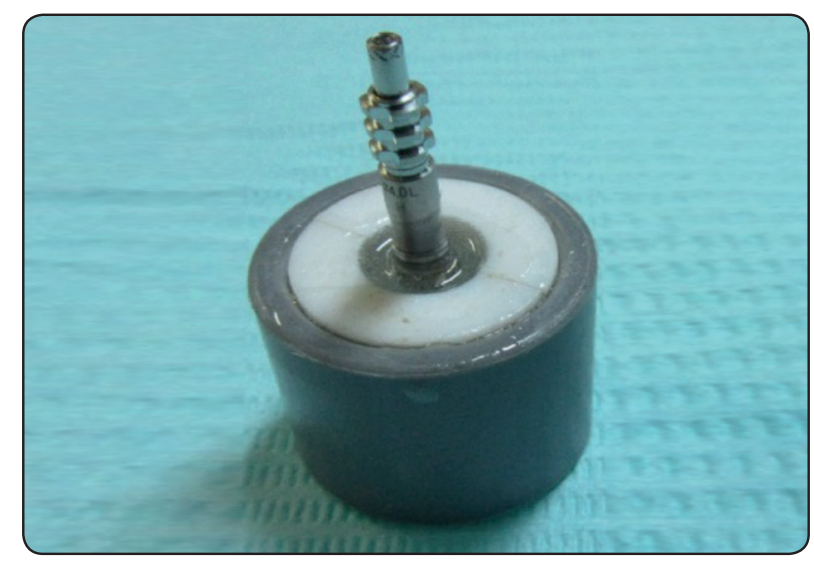

Fig. (1): Implant analog attached to impression copings, mounted vertically in resin block.

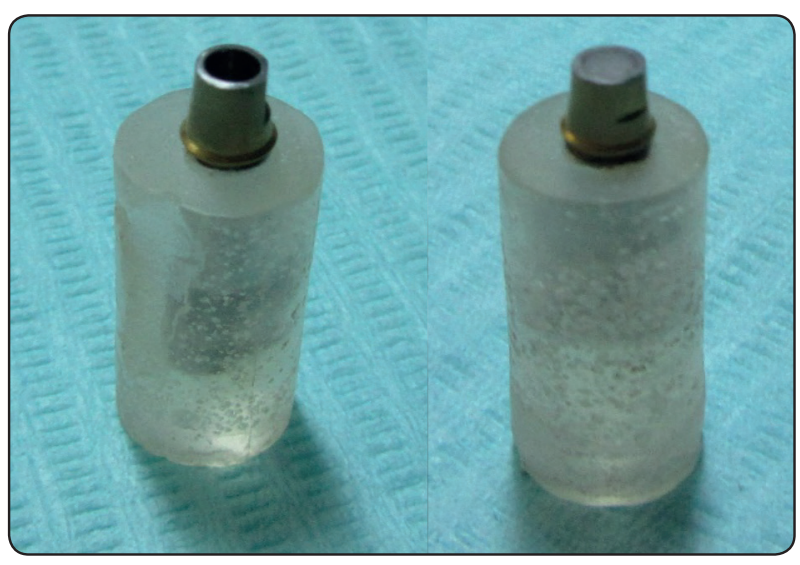

Fig. (2): The abutment was shortened to $4 \mathrm{~mm}$ using diamond disc(a) The screw holes of the abutments were filled with visible light activated composite(b)
All abutments were sprayed with Scan spray (Renfert GmbH Industriegbiet 78247 Hilzingen/ Germany), scanned using Scan Box (Smart Optics Sensortechnik GmbH, Germany) and imported into the CAD/CAM software (InLab version 4.0; Sirona) except f. explore it was milled using (Roland, MDX40; Japan) (Fig.: 3).

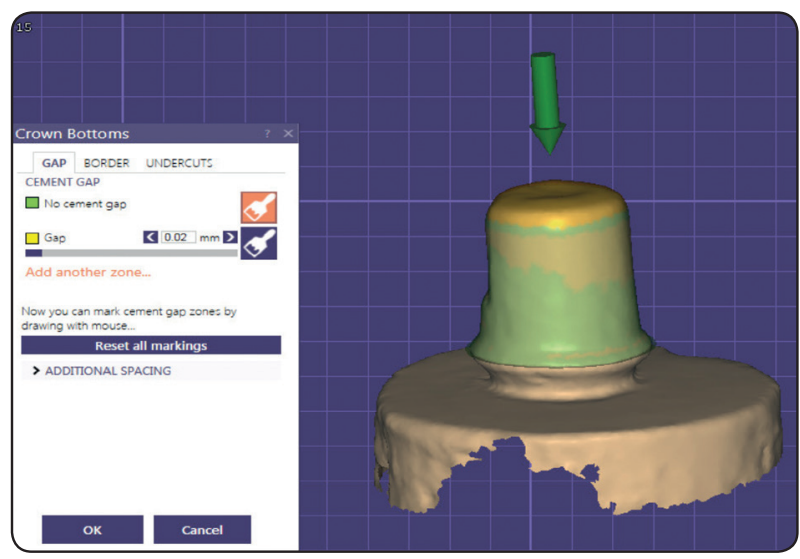

Fig. (3): Scanned abutment.

The ideal anatomical tooth form in EXOCAD Valetta 2.2 software library (Exocad $\mathrm{GmbH}$ ) was used as the reference design, (Fig. 4). Two designs were made to make two groups of crowns (table 1):

Group A $(\mathrm{N}=20)$ : The reference design of anatomical form of lower right first molar with 1.5 $\mathrm{mm}$ thickness at the central fossa.

Group B $(\mathrm{N}=20)$ : The reference design of anatomical form of lower right first molar with 1.0 $\mathrm{mm}$ thickness at the central fossa.

Each group included 4 subgroups, according to the material of which the crows were milled:

Subgroup 1 (N=5): Vita Enamic (V.enamic) (VITA Zahnfabrik, Bad Säckingen, Germany).

Subgroup 2 (N=5): IPS E-max CAD(e.max) (Ivoclar Vivadent, Schaan, Liechtenstein).

Subgroup 3 (N=10): Celtra Duo(CD) (Dentsply DeTrey, Konstanz, Germany)

Subgroup $4 \quad(\mathbf{N}=10)$ : Functional explore (f.explore) (Shenzhen Upcera Dental Technology 


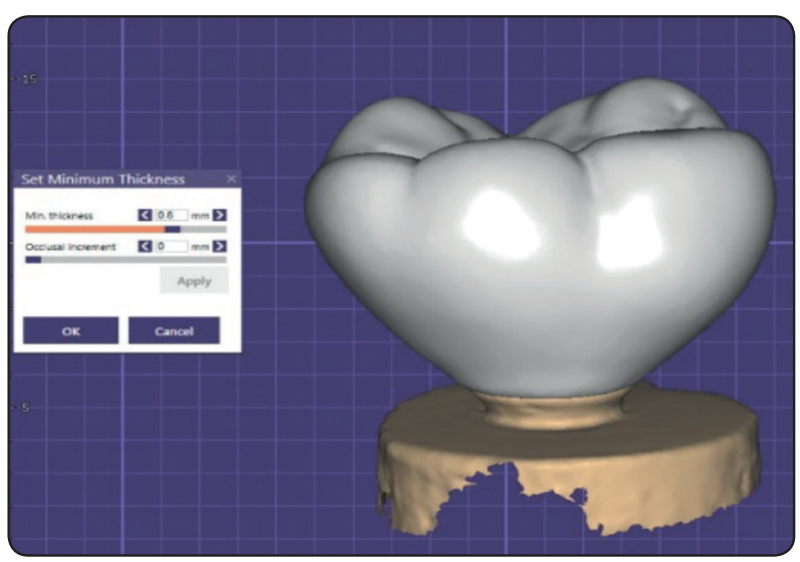

Fig. (4): The design of anatomical tooth form of lower right first molar crown in EXOCAD Valetta 2.2 software.

Co High-tech Industry Park, Nanshan District, Shenzhen, Guangdong, China. Provided by Ivory Trade International, Egypt).

TABLE (1): Samples grouping:

\begin{tabular}{|l|c|c|c|}
\hline & $\begin{array}{c}\text { Group A } \\
(1.5 \mathrm{~mm})\end{array}$ & $\begin{array}{c}\text { Group B } \\
(1.0 \mathrm{~mm})\end{array}$ & \\
\hline $\begin{array}{l}\text { Subgroup 1 } \\
\text { (V.enamic) }\end{array}$ & $\mathrm{N}=5$ & $\mathrm{~N}=5$ & $\mathrm{~N}=10$ \\
\hline $\begin{array}{l}\text { Subgroup 2 } \\
(\mathrm{e}-\text { max })\end{array}$ & $\mathrm{N}=5$ & $\mathrm{~N}=5$ & $\mathrm{~N}=10$ \\
\hline $\begin{array}{l}\text { Subgroup 3 } \\
\text { (CD) }\end{array}$ & $\mathrm{N}=5$ & $\mathrm{~N}=5$ & $\mathrm{~N}=10$ \\
\hline $\begin{array}{l}\text { Subgroup 4 } \\
\text { (f.explore) }\end{array}$ & $\mathrm{N}=5$ & $\mathrm{~N}=5$ & $\mathrm{~N}=10$ \\
\hline \multicolumn{3}{|c|}{ Total } & $\mathrm{N}=40$ \\
\hline
\end{tabular}

For all groups the cement layer thickness was set to $50 \mu \mathrm{m}$. The manufacturer's information of the monolithic ceramics used in this study was shown in Table 2. The design data of the CADbased lower right first molar crowns were used to establish the crowns. Eight experimental groups with two different occlusal thicknesses: $1.5 \mathrm{~mm}$ (recommended condition) and $1.0 \mathrm{~mm}$ (experimental condition).

Four different CAD/CAM ceramic blocks including ; the PICN; Vita Enamic (v.enamic); VITA Zahnfabrik, Bad Säckingen, Germany) finished with rubber (Eve America; Naples, FL); the lithium disilicate; IPS E.max CAD (e.max); (Ivoclar Vivadent, Schaan, Liechtenstein), sintered and finished a with a glaze cycle (Programat P300; Ivoclar Vivadent) ; zirconia- reinforced lithium silicate, Celtra Duo (CD); (Dentsply DeTrey, Konstanz, Germany) Crystallization and glazing process were performed in a furnace (Programat P310; Ivoclar Vivadent) according to the corresponding manufacturer's protocols and f.explore were fabricated into molar-shaped crowns by using four-axis milling machine (Trione G; Dio, Busan,Korea) by Advanced Dental Studio - Egypt. And sintering in ( Tabeo, Mihm Vogt, Germany).

All abutments were sand blasted by $50-\mu \mathrm{m}$ aluminum oxide particles (Renfert, Hilzingen, Germany) at a distance of $10 \mathrm{~mm}$. The specimens were cleaned in distilled water for 3 minutes in ultrasonic bath (Ultrasonic Cleaner; Biem Ultrasonic Makina San. Ltd. Şti., Turkey) in order to remove any surface deposits.

Prior to the cementation, the inner surface of each ceramic crown was treated according to the manufacturer's recommendations. For f.explore group; the intaglio surfaces of the zirconia crowns were air born particle abraded by $50 \mu \mathrm{m} \mathrm{Al}_{2} \mathrm{O}_{3}$ particles at 1 bar pressure then ultrasonically cleaned and air dried.

For CD groups, the inner surfaces of the crowns were washed with ethanol, treated with 5\% hydrofluoric acid gel (Ceramic etching gel; Ivoclar Vivadent) for 20 seconds, and then washed with water. After removing the residual acid with water, they were air-dried and coated with silane (Monobond N; Ivoclar Vivadent).

For v.enamic group, the inner surfaces were washed with ethanol, treated with $5 \%$ hydrofluoric acid gel (Ceramic etching gel; Ivoclar Vivadent) for 60 seconds, and then washed with water for 60 seconds. Afterwards, they were air-dried for 20 seconds and coated with silane (Monobond N; Ivoclar Vivadent). 
TABLE (2): All-ceramic crown materials used in the study

\begin{tabular}{|c|c|c|}
\hline Material & Description, properties \& composition & Manufacturer \\
\hline $\begin{array}{l}\text { hybrid ceramic } \\
\text { (Vita Enamic) } \\
\text { (groupI) }\end{array}$ & $\begin{array}{l}\text { Double network hybrid ceramic block, Shade 3M2-HT EM-14 } \\
\text { Flexural strength : } 150-160 \mathrm{MPa} \\
\text { Fracture toughness: } 1.5 \mathrm{MPa} \mathrm{m}^{1 / 2} \\
\text { Elastic modulus : } 30 \mathrm{GPa} \\
\text { Composition of the ceramic part : (86 wt\% / } 75 \text { vol\%) } \\
\text { Silicon dioxide } \mathrm{SiO} 258-63 \% \\
\text { Aluminum oxide } \mathrm{Al} 2 \mathrm{O} 320-23 \% \\
\text { Sodium oxide } \mathrm{Na} 2 \mathrm{O} 9-11 \% \\
\text { Potassium oxide } \mathrm{K} 2 \mathrm{O} 4-6 \% \\
\text { Boron trioxide } \mathrm{B} 2 \mathrm{O} 30.5-2 \% \\
\text { Zirconium dioxide } \mathrm{ZrO} 2<1 \% \\
\text { Calcium oxide } \mathrm{KaO}<1 \% \\
\text { Composition of the polymer part : (14 wt } \% \text { / } 25 \text { vol\%) } \\
\text { UDMA (urethane dimethacrylate) TEGDMA (triethylene glycol dimethacrylate) }\end{array}$ & $\begin{array}{l}\text { VITA Zahnfabrik } \\
\text { Germany }\end{array}$ \\
\hline $\begin{array}{l}\text { (group II) } \\
\text { Lithium- } \\
\text { disilicate glass } \\
\text { ceramic } \\
\text { (IPS e. max } \\
\text { CAD) } \\
\text { (group II) }\end{array}$ & $\begin{array}{l}\text { Lithium disilicate glass-ceramic block } \\
\text { Flexural strength : } 360 \pm 60 \mathrm{MPa} \\
\text { Fracture toughness: } 2.0-2.5 \\
\text { Elastic modulus : } 95 \pm 5 \mathrm{GPa} \\
\text { Composition in } W t \%: \\
\text { Silicon dioxide } \mathrm{SiO} 257.0-80.0 \\
\text { Lithium dioxide } \mathrm{Li} 2 \mathrm{O} 11.0-19.0 \\
\text { Potassium oxide } \mathrm{K} 2 \mathrm{O} 0.0-13.0 \text { Phosphorus pentoxide } \mathrm{P} 2 \mathrm{O} 50.0-11.0 \\
\text { Zirconium dioxide } \mathrm{ZrO} 20.0-8.0 \\
\text { Zinc oxide } \mathrm{ZnO} 0.0-8.0 \\
\text { Other \& coloring oxides } 0.0-12.0\end{array}$ & $\begin{array}{l}\text { Ivoclar Vivadent } \\
\mathrm{S} \text { c h a a n, } \\
\text { Liechtenstein }\end{array}$ \\
\hline $\begin{array}{l}\text { Zirconia- } \\
\text { reinforced } \\
\text { Lithium Silicate } \\
\text { (ZLS)Celtra duo }\end{array}$ & $\begin{array}{l}10 \% \text { zirconium dioxide }(\mathrm{ZrO} 2) \text { in highly dispersed form in the glass phase of the } \\
\text { ceramic blocks. } \\
\text { Flexural strength : } 370 \mathrm{MPa} \\
\text { Fracture toughness : } 2.56 \mathrm{MPa} \mathrm{m}^{1 / 2} \\
\text { Elastic modulus : } 70 \mathrm{GPa} \\
\text { Composition of the ceramic part } \\
\text { Zirconia } 10 \% \\
\text { Silica, Lithium metasilicate and phosphate crystals } 58 \%\end{array}$ & $\begin{array}{l}\text { Dentsply DeTrey, } \\
\text { Konstanz, Germany }\end{array}$ \\
\hline $\begin{array}{l}\text { Yttria } \\
\text {-stabilized } \\
\text { tetragonal } \\
\text { zirconia } \\
\text { polycrystalline } \\
\text { (Upcera ) } \\
\text { (group VI) }\end{array}$ & $\begin{array}{l}\text { Upcera Zirconia , specialized for Full Contour Zirconia restorations } \\
\text { Multi Layered Zirconia disc with } 98.5 \mathrm{~mm} \text { diameter and } 18 \\
\text { mm thickness, shade A light } \\
\text { Flexural strength : } 1125 \mathrm{MPa} \\
\text { Fracture toughness :5.1 } \mathrm{MPa} \mathrm{m}^{1 / 2} \\
\text { Elastic modulus : } 214 \mathrm{GPa} \\
\text { Composition in } \mathbf{W t} \% \text { : } \\
\text { Zirconium dioxide } \mathrm{ZrO} 2+\mathrm{HfO} 290-95 \% \\
\text { Yttrium Oxide } \mathrm{Y} 2 \mathrm{O} 35-8 \% \\
\text { Other oxides } \mathrm{CaO}, \mathrm{MgO} 0-2 \%\end{array}$ & $\begin{array}{l}\text { Shenzhen Upcera } \\
\text { Dental Technology } \\
\text { Co, China }\end{array}$ \\
\hline
\end{tabular}


For e. max group, the inner surfaces were treated with 5\% hydrofluoric acid gel (Ceramic etching gel; Ivoclar Vivadent) for 20 seconds, washed with water and air-dried, and coated with silane coupling agent (Monobond N; Ivoclar Vivadent).

Subsequently, self-adhesive resin cement (RelyX U200; 3M ESPE) was used to bond the crowns to the abutments according to the manufacturer's protocol.

The crowns were seated onto the implant abutment with finger pressure and then $5 \mathrm{~kg}$ were applied onto each crown using a specially designed loading device, for 10 minutes. Cement excess was removed with a micro brush and each surface was light-cured for $40 \mathrm{~s}$ with a LED curing unit (Elipar S10, 3M ESPE, Seefeld, Germany).

The bonded crown/abutment specimens were stored in distilled water at $37^{\circ} \mathrm{C}$ for 24 hours prior to thermal and mechanical cycling. ${ }^{5}$

Thermo-cycling Mechanical loading (TCML) was performed using a programmable equipment; the newly developed four stations multimodal ROBOTA chewing simulator integrated with thermo-cyclic protocol operated on servo-motor (Model ACH09075DC-T, AD-TECH TECHNOLOGY CO., LTD., GERMANY) ROBOT. A chewing simulator which has four chambers simulating the vertical and horizontal movements simultaneously in the thermodynamic condition. Each of the chambers consists of an upper Jackob's chuck as hardened steel antagonist holder that can be tightened with a screw and a lower plastic sample holder in which the specimen can be embedded. The specimens were embedded in chemical cured acrylic mold which in turn fixed by tightening screw to teflon holder in the lower part of simulator. A weight of $5 \mathrm{~kg}$, comparable to $49 \mathrm{~N}$ of chewing force was exerted. The test was repeated 75,000 times to clinically simulate the 6 months chewing condition, according to previous studies. ${ }^{(18)}$ Chewing simulation test parameters
Vertical movement: $3 \mathrm{~mm}$. Horizontal movement: $1 \mathrm{~mm}$. Rising speed: $90 \mathrm{~mm} / \mathrm{s}$. Forward speed: $90 \mathrm{~mm} / \mathrm{s}$. Descending speed: $40 \mathrm{~mm} / \mathrm{s}$. Backward speed: $40 \mathrm{~mm} / \mathrm{s}$. Cycle frequency $1.6 \mathrm{~Hz}$ Weight per sample: 5 kg Torque; 2.4 N.m. During TCML all crowns were controlled daily for failures and failed crowns were excluded from further simulation and testing.

\section{Fracture resistance testing}

All samples were individually mounted on a computer-controlled material testing machine (Model 3345; Instron Industrial Products, Norwood, MA, USA) (Fig. 5) with $5 \mathrm{kN}$ load cell and data were documented using computer software (Instron ${ }^{\circledR}$ Bluehill Lite Software). Samples were secured to the lower fixed compartment of testing machine by tightening screws. Fracture test was done by compressive mode of load applied occlusally using a metallic rod with spherical tip (5.8 $\mathrm{mm}$ diameter) attached to the upper movable compartment of testing machine traveling at cross-head speed of $1 \mathrm{~mm} / \mathrm{min}$ with tin foil sheet in-between to achieve homogenous stress distribution and minimization of the transmission of local force peaks. The load required to fracture was recorded in Newton. After the testing, the fractured surface of each specimen

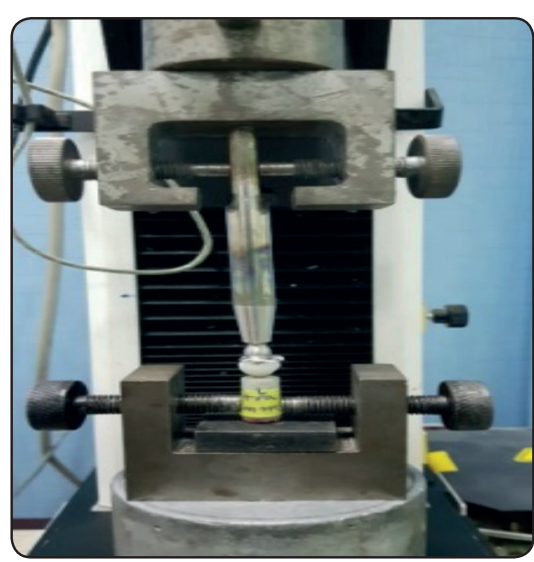

Fig. (5): sample mounted on a computer-controlled material testing machine. 
was observed under a stereomicroscope at a magnification of (36-50X), and one representative specimen from each group was randomly selected for the fractographic analysis. The fractographic analysis was conducted to examine the fracture characteristics of the various ceramic crowns and to identify the crack propagation direction, under a scanning electron microscope (SEM) (JEOL JSMT 20. Japan) operating at $20.0 \mathrm{Kv}$, at (36-50X) for fracture pattern examination.

\section{Statistical analysis}

The results were analyzed using Graph Pad Instat (Graph Pad, Inc.) software for windows. A value of $\mathrm{P} \leq 0.05$ was considered statistically significant. Continuous variables were expressed as the mean and standard deviation. After homogeneity of variance and normal distribution of errors had been confirmed, Analysis of variance was used to compare materials. Student t-test was done for compared pairs. Two-factors analysis of variance for each factor. Sample size $(n=5)$ was large enough to detect large effect sizes for main effects and pairwise comparisons, with the satisfactory level of power set at $80 \%$ and a $95 \%$ confidence level.

\section{RESULTS}

\section{Fracture resistance}

The results of fracture resistance $(\mathrm{N})$ mean, standard deviation (SD) values for all groups with both occlusal thickness after mechanical aging are summarized in table (3) and graphically drawn in figure (6).

\section{$1 \mathrm{~mm}$ occlusal thickness}

The highest fracture resistance mean value recorded with f.explore group (3951 N) followed by CD group $(1356.2 \mathrm{~N})$ followed by e.max group $(1008.6 \mathrm{~N})$ while the lowest fracture resistance mean value recorded with V. Enamic group (811.7 $\mathrm{N})$ and this was statistically significant as indicated by one-way ANOVA followed by Tukey's pair-wise tests $(\mathrm{P}=<0.0001<0.05)$, as shown in table $(3)$ and figure (6).

\section{$1.5 \mathrm{~mm}$ occlusal thickness}

It was found that the highest fracture resistance mean value recorded with f.explore group $(4663 \mathrm{~N})$ followed by Celtra group $(1617 \mathrm{~N})$ then e.max group $(962.23 \mathrm{~N})$ while the lowest fracture resistance

TABLE (3): Fracture resistance results (Mean values in Newton \pm SDs) for all groups with both occlusal thicknesses.

\begin{tabular}{|c|c|c|c|c|c|}
\hline \multirow{2}{*}{\multicolumn{3}{|c|}{ Variables }} & \multicolumn{2}{|c|}{ Occlusal thickness } & \multirow{2}{*}{$\frac{\text { t-test }}{\text { P value }}$} \\
\hline & & & $1 \mathrm{~mm}$ & $1.5 \mathrm{~mm}$ & \\
\hline \multirow{9}{*}{ Material type } & \multirow{2}{*}{ V. Enamic } & Mean & $811.7^{D}$ & $891.62^{C}$ & \multirow{2}{*}{$0.1914 \mathrm{~ns}$} \\
\hline & & $S D$ & \pm 54.58 & \pm 69.205 & \\
\hline & \multirow{2}{*}{ e.max } & Mean & $1008.6^{C}$ & $962.23^{C}$ & \multirow{2}{*}{$0.6488 \mathrm{~ns}$} \\
\hline & & $S D$ & \pm 98.45 & \pm 130.4 & \\
\hline & \multirow{2}{*}{ Celtra } & Mean & $1356.2^{B}$ & $1617^{B}$ & \multirow{2}{*}{$0.1221 \mathrm{~ns}$} \\
\hline & & $S D$ & \pm 152.1 & \pm 173.8 & \\
\hline & \multirow{2}{*}{ f.explore } & Mean & $3951^{A}$ & $4663^{A}$ & \multirow{2}{*}{$0.0432 *$} \\
\hline & & $S D$ & \pm 227.225 & \pm 356 & \\
\hline & ANOVA & $P$ value & $<0.0001 *$ & $<0.0001^{*}$ & \\
\hline
\end{tabular}

Different letters in same column indicating significant $(p<0.05) \quad *$; significant $(p<0.05)$ 


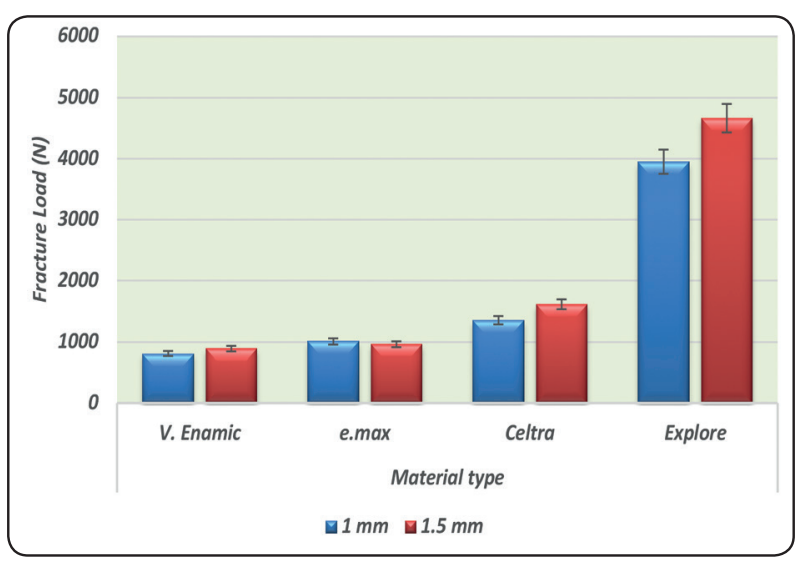

Fig. (6) Column chart of fracture load mean values for all groups with both occlusal thicknesses.

mean value recorded with V. Enamic group (891.62 $\mathrm{N})$ and this was statistically significant as indicated by one-way ANOVA test $(\mathrm{P}=<0.0001<0.05)$. Pairwise Tukey's post-hoc test showed non-significant $(\mathrm{p}>0.05)$ difference between (V. Enamic and e.max) as shown in table (3) and figure (6)

\section{$1 \mathrm{~mm}$ vs. $1.5 \mathrm{~mm}$ occlusal thickness}

For V. enamic group; $1.5 \mathrm{~mm}$ occlusal thickness recorded statistically non-significant higher fracture resistance mean value $(891.6 \mathrm{~N})$ than $1 \mathrm{~mm}$ occlusal thickness type mean $(811.7 \mathrm{~N})$ as proved by paired $\mathrm{t}$-test $(\mathrm{p}=0.1914>0.05)$.

E.max group: $1 \mathrm{~mm}$ occlusal thickness recorded statistically non-significant higher fracture resistance mean value $(1008.6 \mathrm{~N})$ than $1.5 \mathrm{~mm}$ occlusal thickness type mean $(962.23 \mathrm{~N})$ as proved by paired t-test $(\mathrm{p}=0.6488>0.05)$

CD group: $1.5 \mathrm{~mm}$ occlusal thickness recorded statistically non-significant higher fracture resistance mean value $(1617 \mathrm{~N})$ than $1 \mathrm{~mm}$ occlusal thickness type mean $(1356.2 \mathrm{~N})$ as proved by paired $\mathrm{t}$-test $(\mathrm{p}=0.1221>0.05)$.

F.explore group: $1.5 \mathrm{~mm}$ occlusal thickness recorded statistically significant higher fracture resistance mean value $(4663 \mathrm{~N})$ than $1 \mathrm{~mm}$ occlusal thickness type mean $(3951 \mathrm{~N})$ as proved by paired t-test $(\mathrm{p}=0.0432<0.05)$.

\section{Influence of material group on fracture resistance}

Regardless to cementation approach, totally it was found that the highest fracture resistance was recorded with f.explore group followed by $\mathrm{CD}$ group then e.max group while the lowest fracture resistance mean value recorded with v. Enamic with statistical significance as indicated by two-way ANOVA test $(\mathrm{P}=0.0001<0.05)$. Pair-wise Tukey's post-hoc test showed non-significant $(p>0.05)$ difference between (e.max and v. enamic) .

\section{Effect of occlusal thickness on fracture resistance}

Irrespective of material groups, totally it was found that $1.5 \mathrm{~mm}$ occlusal thickness recorded statistically non-significant higher fracture resistance mean value than $1 \mathrm{~mm}$ occlusal thickness except for f.explore group as demonstrated by twofactors ANOVA test $(\mathrm{P}=0.2267>0.05)$.

\section{Scanning Electron Microscope analysis}

The failure mode of the samples was a combination of fracture and debonding, some of the f.explore crowns with occlusal thickness of 1.5 $\mathrm{mm}$ were not fractured even at $10 \mathrm{kN}$ and associated with abutment fracture.

The SEM image of the fractured surface showed multiple cracks together with arrest lines, some compression curls, and twist hackle marks (Figs. 7 to 10). The origin of fracture seems to arise at the occlusal surface of the E.max and Enamic crowns, while for $\mathrm{f}$. explore and $\mathrm{CD}$ groups the fracture seems to arise from the intaglio surface of the crowns. For the Enamic crowns, the cracks were shown to arise from the occlusal surface and then end before reaching the fitting surface. For the Emax crown, a tuft of cracks were arising from the loading point at the occlusal surface. For the f.explore and CD crowns, a main fracture line seems to arise from the fitting surface of the crown and the cracks extend to the outer proximal and cervical area. 


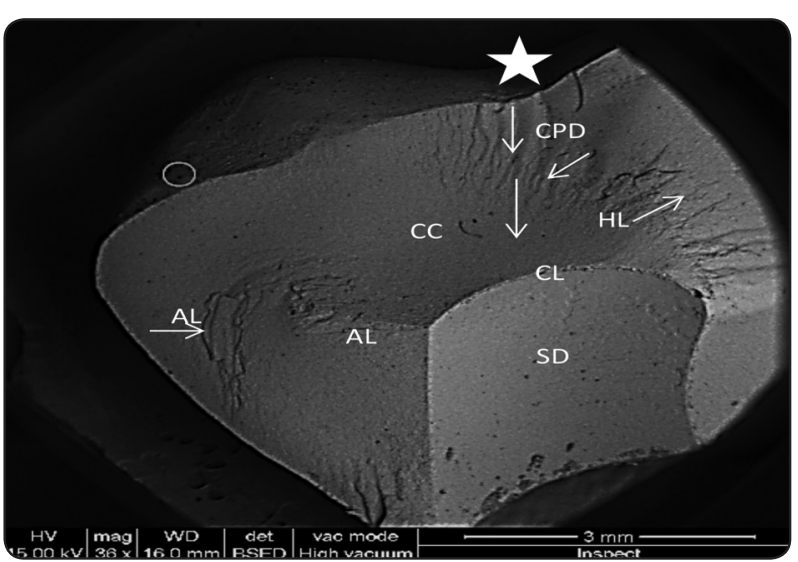

Fig. (7): SEM image of the fractured surface of v. enamic crown (group A1) with occlusal thickness of $1.5 \mathrm{~mm}$. hackle line (HL), arrest line (AL), the star: where the fracture began, the arrows: the direction where cracks propagated, $\mathrm{CC}$ : compression curle (CPD): crack propagation direction, (CL) cementation layer, and (SD) supporting die.

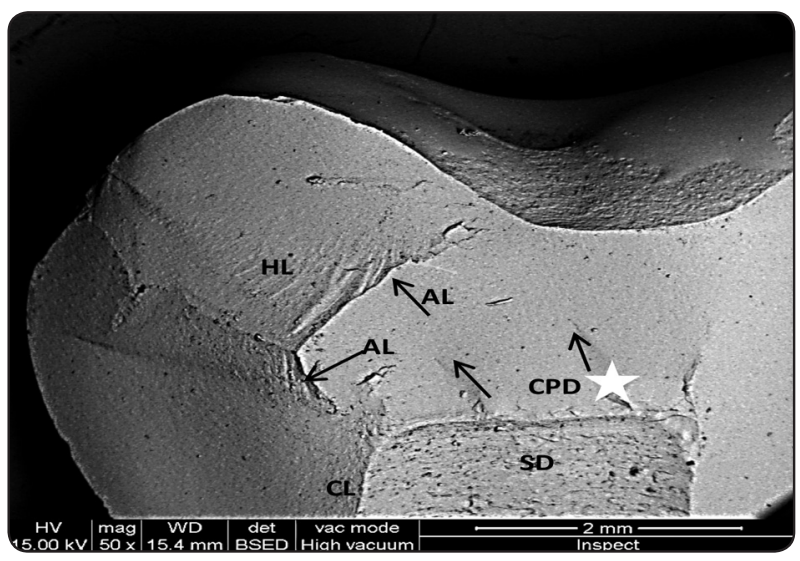

Fig. (9): SEM image of the fractured surface of CD crown with occlusal thickness of $1.5 \mathrm{~mm}$. HL: hackle line, AL: arrest line, CC: compression curl, TH: twist hackle, white star: origin of fracture, black solid arrow: crack propagation direction (CPD), CL: cementation layer, SD: supporting die.

\section{DISCUSSION}

Implant-supported crowns gained popularity as they were proved to have good clinical survival rate in long-term studies. ${ }^{(19)}$ Specially when used with monolithic ceramic crowns showing aesthetic improvement above metal ceramic restorations, with adequate mechanical properties. ${ }^{(20)}$ However, occlusal fractures occasionally were reported in clinical practice specifically with inadequate inter-

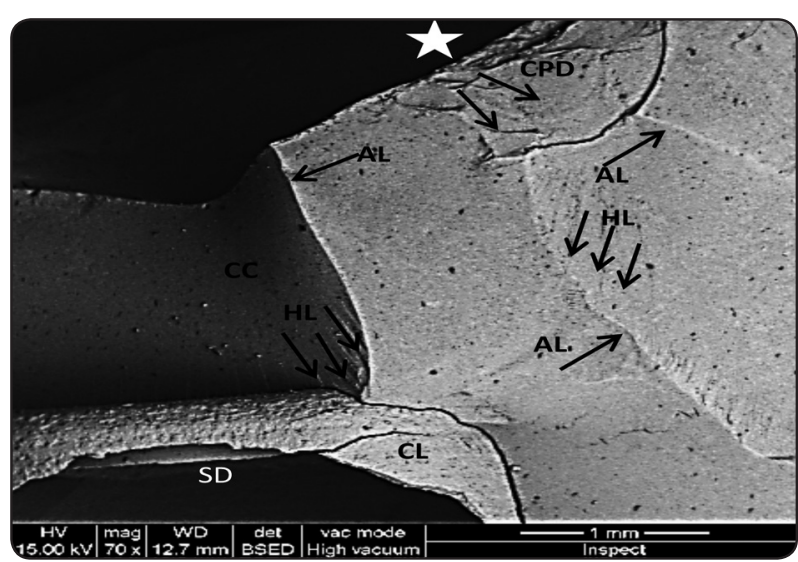

Fig. (8): SEM image of the fractured surface of e. max crown with occlusal thickness of $1.5 \mathrm{~mm}$. HL: hackle line, AL: arrest line, the star: where the fracture began, TH: twist hackle, black solid arrow: crack propagation direction (CPD), CL: cementation layer, SD: supporting die

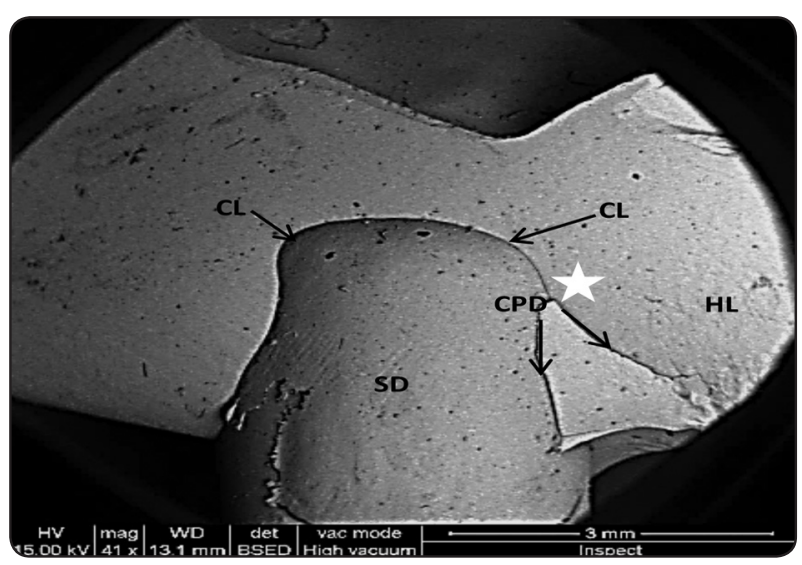

Fig. (10): SEM image of the fractured surface of f.explore crown with occlusal thickness of $1 \mathrm{~mm}$ with deeply located crack origin from the interface between the crown and cement layer.

arch space. This study evaluated four monolithic implant-supported crowns machined with CEREC system using the mandibular first molar as a reference.

In the present study, the CAD/CAM system was chosen, as it allowed for the use of high-quality materials, such as CAD/CAM-prefabricated blocks and this system also allowed for the standardization of manufactured crowns. In the monolithic 
restorations there is no further veneering layer, and hence the construction is simplified. ${ }^{(21)}$

Posterior restorations are subjected to high masticatory forces. The reported maximum biting forces in posterior region vary from the 600 to 900 $\mathrm{N}$. The variations were shown to be influenced by facial morphology and age.[22]. In this study, the force needed to fracture the crowns surpassed the maximum biting force in the molar region regardless of their occlusal thickness, which means that all four materials tested are recommended for cemented implant-supported crowns.

Since the fracture resistance is influenced by the occlusal thickness of ceramic crowns, It was recommended to achieve $1.3 \mathrm{~mm}$ to $2.0 \mathrm{~mm}$ thickness at the occlusal surface of ceramic crowns. $(23,24)$ This study assumed that clinical situations may dictate the use of $1.0 \mathrm{~mm}$ occlusal thickness; therefore ceramic crowns with $1.5 \mathrm{~mm}$ as generally accepted, as well as $1.0 \mathrm{~mm}$ were tested.

The standardization in the present study was simplified by using implant abutments because in natural teeth standardization regarding to properties and forms could be problemist.

In the current study, aging of the samples was done by using chewing simulator to exert intermittent occlusal equivalent to chewing force together with thermos-cycling. The load was repeated 75.000 times to clinically simulate a six months chewing condition, according to previous studies. ${ }^{(18)}$

Temperature changes and exposure to water were found to distress the strength properties of ceramic restorations, ${ }^{(25)}$ and static fatigues with slow flaw propagation were reported. ${ }^{(26)}$ Thus, samples of this study were subjected to thermocycling to simulate the clinical situation as carry out by many authors. ${ }^{(25-27)}$ Dynamic fatigue was shown to decrease fracture resistance of several materials including dental ceramics that can fracture during function.

In the existent study crowns were cemented using self-adhesive resin cement (RelyX U200; 3M
ESPE). This shortened delivery procedure makes it clinically applicable, fast, and appreciated by clinicians.

The results of this study showed that; the fracture resistance of monolithic crowns was differently affected by the type of ceramic material; therefore, the first null hypothesis is rejected. F.explore group verified the highest fracture resistance followed by $\mathrm{CD}$ group then e.max group whereas the lowest fracture resistance means value were verified for v.enamic group when the occlusal thickness was identical . However, differences between the four materials fracture resistance can be ascribed to the material structure and the mechanical properties (Table 2). ${ }^{(28)}$ The outcomes of the current study were in accordance with those of Kok et al ${ }^{(29)}$ who examined the mechanical behavior of different implant-supported posterior crowns and found that, the highest initial load to failure was for anatomic contour zirconia crowns followed by e. max CAD then Vita enamic. The results were also in agreement with those of Kim et al ${ }^{(30)}$ who reported that implant supported zirconia posterior crowns had significantly higher fracture resistance comparing to e. max CAD crowns.

Interestingly, the present study displayed that an increase in occlusal thickness from 1 to $1.5 \mathrm{~mm}$ didn't affect the fracture resistance for all material used except the f.explore group, therefore the second null hypothesis is partly rejected. F.explore group with $1.5 \mathrm{~mm}$ occlusal thickness has significantly higher fracture resistance than that with $1.0 \mathrm{~mm}$ occlusal thickness, this comes in consistence with Nakamura $\mathrm{N}$ et $\mathrm{al}^{(31)}$ who found that the monolithic zirconia crowns strength was dependent on the occlusal thickness. Moreover, in the load-to-failure test, some of the f.explore crowns with occlusal thickness of $1.5 \mathrm{~mm}$ were not fractured even at $10 \mathrm{kN}$. This result is consistent with a previous report wherein Beuer et al. ${ }^{(32)}$ demonstrated that 11 out of 12 monolithic zirconia crowns did not fail at $10.5 \mathrm{kN}$. This could be attributed to the special formulation of $\mathrm{f}$. explore zirconia blanks which consist of 5 layers having 
different types of zirconia powder within the same blank; $1^{\text {st }}$ layer $(4 \mathrm{Y}-\mathrm{TZP}), 2^{\text {nd }}, 3^{\text {rd }}$, and $4^{\text {th }}$ layers are the combination of (4Y-TZP) and (3Y-TZP) so that each layer has different portions of (4Y-TZP) and (3-TZP), therefor different combination of strength and translucency. The $5^{\text {th }}$ layer is (3Y-TZP). ${ }^{(33)}$

In this study, v.enamic crowns required the least load to fracture $(891 \mathrm{~N})$. This comes in accordance with the relatively low mechanical properties of this material including low flexural strength (150-160 $\mathrm{MPa}$ ) and low fracture toughness (1.5 MPa). It can be explained by the hybrid nature of this material as it is composed of interconnected networks of ceramic and polymer, the different behavior of ceramic and polymer during the grinding and polishing processes may result in micro-cracks in the network boundaries, and this is assumed to affect the mechanical properties of the material ${ }^{(34}$, ${ }^{35)}$. Moreover, it was shown that, in a hybrid material, failure can be initiated from any weak point of the microstructure, like the polymer in polymer infiltrated ceramic ${ }^{(36)}$. This comes in accordance with the findings of Bilkhair ${ }^{27}$ who had a study to compare the fracture strength of monolithic hybrid ceramic crowns with lithium disilicate and feldspathic ceramic crowns. In his study it was shown that the hybrid dental ceramic crowns had lower fracture resistance than that for lithium disilicate crowns. In other study by Sieper et al. ${ }^{(37)}$ that compared the fracture strength of hybrid dental ceramic, lithium disilicate and zirconia-reinforced lithium silicate. It was shown that hybrid ceramic crowns recorded the lowest fracture strength among the tested materials.

Moreover, the outcomes of this study presented that occlusal thickness variations in v.enamic crowns had none significant influence its fracture resistance. The results comes in consistence with the work of Choi S et al. ${ }^{38}$ and Chen $\mathrm{C}$ et al ${ }^{39}$; the later studied a standardized molar onlays using resin nano-ceramics and they found no significant difference in fracture resistance between 1.0 $\mathrm{mm}$ and $1.5 \mathrm{~mm}$ occlusal thickness. The authors advocated that difference in mechanical properties of resin surrounding substance and ceramic particles created fracture resistance comparative to thickness variations inside a certain range. Similarly, the stress distribution and fracture manner of v.enamic was dissimilar from glass ceramics owing to elastic polymer complex. Also this result was in accordance with Sallam H. who found that V. enamic crowns on implant abutments used recorded the lowest fracture resistance among the tested ceramic crowns. The author explained this result as; hybrid ceramics are not as strong as ceramics. ${ }^{(40)}$ Vita enamic is based on a network structure made of aluminum oxideenriched, fine structure feldspar ceramic combined with a proportion of polymer material consisting of UDMA and TEG-DMA. However, its lower modulus of elasticity made it a suitable material for implant supported restorations. ${ }^{(41-43)}$

On the other hand, according to the current study; it was found that an increase in occlusal thickness of v.enamic to $1.5 \mathrm{~mm}$ lead to fracture resistance increase to be insignificantly different from e.max at the same occlusal thickness. This result is agreed with Menini M and Conserva E. ${ }^{(43)}$, they have explained that resin-based materials and composites have higher shock absorbing capacity than ceramics. $(20,21)$ This was in agreement with findings by Kok et al. ${ }^{(29)}$ who found higher fracture values for materials with a lower modulus of elasticity. On the other side, the results were contradicting with those of Rosentritt et al ${ }^{(44)}$ and Weyhrauch et al ${ }^{(45)}$ who tested the fracture resistance of several CAD/CAM fabricated all ceramic implant supported posterior crowns and found that IPS e max CAD crowns registered statistically significant higher fracture resistance values than v.enamic crowns at $1.5 \mathrm{~mm}$ occlusal thickness.

E.max and CD crowns recorded lower mean values of fracture strength than f.explore crowns with highly significant differences. This might be due to the characteristic lower mechanical properties as flexural strength, lower elastic moduli and lower fracture toughness of the two materials comparing 
with zirconia. The difference in occlusal thickness in the LD group had no significant effect on fracture resistance. This came in accordance with the work of Sorrentino $\mathrm{R}$ et al ${ }^{\left({ }^{46)}\right.}$ who found that LD crowns with $1 \mathrm{~mm}$ occlusal thickness showed higher fracture resistance than 0.5 and 1.5 occlusal thickness; consequently, in agreement with the manufacturer's instructions, the author stated that it is possible to design posterior LD crowns with $1 \mathrm{~mm}$ occlusal thickness to guarantee the greatest mechanical performances under function. They attributed these results to the fact that, with less bulk of ceramic the stresses are transmitted and absorbed by the resin cement, on the other hand, the stress adsorbing capability of resin cements could not be entirely effective in the presence of bulk thicknesses of glass ceramics and this mechanical drawback could lead to intrinsic micro cracks of lithium disilicate causing clinical failures. This result comes in consistence with Sieper k et al ${ }^{(47)}$ and Sydler B et al ${ }^{(48)}$ who demonstrated that an occlusal thickness of $1.0 \mathrm{~mm}$ allowed monolithic lithium disilicate crowns to withstand occlusal forces in the molar areas. On the other hand, this result was contradicted with Choi S et al ${ }^{(50)}$; who's displayed a direct relation between thickness and fracture load.

Results of the present study found that CD group recorded higher statistically difference fracture strength than e.max group this came in harmony with the results Of Preis et al. ${ }^{51}$, Schwindling et al. ${ }^{52}$ and Jassim $\mathrm{Z} \mathrm{M}^{28}$ et al. the authors attributed this to the following reasons; first: The diffusion of nano- zirconia particles in the CD glassy matrix (10\%), looks to improve the fracture resistance comparing with e.max. The integrated zirconia particles boosted the production of a larger amount of minor crystallites $(0.5-1 \mu)$ rather than the smaller number of large crystallites $(1.5 \mu)$ that are existent in the e.max, and this comes in accordance with microstructure appearance which demonstrates that the glass part of CD is present at a higher ratio comparing with conventional LD in spite of LD has higher percentage of crystal part (about $70 \%$ ) comparing to C D (40-50\%) ${ }^{(53)}$. At the same time, the integrated zirconia will undergo phase transformation and increase the fracture toughness by preventing crack propagation ${ }^{54}$. This explains the outcomes of a SEM of this study which displayed distinct crescent arrest lines nearby the beginning of failure were presented in CD crowns, which makes the supposition that micro cracks may have an effect on the fracture strength of $\mathrm{CD}$ comparing with LD crowns which display leading hackles from the beginning of failure toward the die ${ }^{(55)}$. Second: The lower modulus of elasticity of CD (70 GPa) comparing with e.max (95 GPa), which recommends that stress collected in the e.max crowns is more than that collected in CD crowns. However, the above outcome differs from the results of Sieper et al. ${ }^{47}$ Gungor and Nemli ${ }^{56}$ whom experienced the fracture resistance of crowns madeup from LD, zirconia reinforced lithium silicate and other ceramic materials and found that the fracture resistance of $\mathrm{LD}$ was higher than zirconia-reinforced lithium silicate crowns. Such disagreement may be due to the difference in the type of zirconiareinforced lithium silicate material used as they used Vita Suprinity. This was contradicted with Choi S. et al ${ }^{(50)}$ whereas the CD group exhibited a significant lower value comparing with e.max and alterations in the restoration thickness significantly affect the $\mathrm{CD}$ fracture resistance.

The mean fracture resistance of all tested ceramic groups in this study went from $811 \mathrm{~N}$ to $4663 \mathrm{~N}$. This advocated that all the monolithic crowns used were acceptable for clinical usage because they can experimentally resist the average $(700 \mathrm{~N})$ or the maximum physiological forces $(1000 \mathrm{~N})$ exercised on posterior teeth. ${ }^{(57,58)}$

The v. enamic. and LD crowns in the existing study displayed that the fractures started in the load contact area, protracted to the cervical area, and lead to complete crown separation. In the v. enamic. sample, the spreading of minor crack was stopped within the deep occlusal surface, signifying that possible crack limitation due to respectable damage tolerance. On the contrary, the $\mathrm{CD}$ and f.explore crowns presented dissimilar forms of fractures, with deeply located crack starts near the 
interfaces between the crown and cement layer. The compression curls and twisted hackle results with bifurcations advocated probable crack propagation across the occlusal surface. As all the tried crowns were destructed catastrophically.

\section{CONCLUSIONS}

Within the restrictions of this study, the subsequent conclusions could be withdrawn:

1- Occlusal thickness significantly affects the fracture resistance of f.explore zirconia groups, while it has non-significant effect for all the other groups.

2- F.explore group with $1.5 \mathrm{~mm}$ occlusal thickness has significantly higher fracture resistance than that with $1.0 \mathrm{~mm}$ occlusal thickness, and both have significantly higher fracture resistance than all the other groups.

3- Celtra duo groups have significantly higher fracture resistance than IPS E-max CAD and Vita Enamic groups.

4- IPS E-max CAD groups have non-significantly higher fracture resistance than Vita Enamic group with $1.5 \mathrm{~mm}$ occlusal thickness.

5- Vita Enamic group with $1.0 \mathrm{~mm}$ occlusal thickness has significantly lower fracture resistance than all the other groups.

\section{ACKNOWLEDGEMENT}

The author would like to thank Ivory Trade International Co. the dealer for Upcera Company in the Egyptian market for supplying Functional Explore zirconia disks for this research in coordination with Advanced Dental Studio dental lab.

\section{REFERENCES}

1. Anadioti E, Aquilino SA, Gratton DG, Holloway JA, Denry IL, computer-aided manufacturing ceramic crowns made from digital and conventional impressions. J Prosthet Dent. 2015;113(4):304-9.

2. Baldissara P, Llukacej A, Ciocca L, Valandro FL, Scotti R. Translucency of zirconia copings made with different
CAD / CAM systems. J Prosthet Dent. 2010;104(1):6-12.

3. Borba M, Miranda WG Jr, Cesar PF, Griggs JA, Della Bona A. Evaluation CT technology. Braz Oral Res. 2013;27(5):396-402.

4. Campos RE, Soares $\mathrm{P}$ V, Versluis A, Júnior OB, Ambrosano GM, Nunes IF. Crown fracture: failure load, stress distribution, and fractographic analysis. J Prosthet Dent. 2015;114(13):447-55.

5. Pjetursson BE, Sailer I, Zwahlen M, Hämmerle CH. A systematic review of the survival and complication rates of all ceramic and metal-ceramic reconstructions after an observation period of at least 3 years. Part I: Single crowns . Clin OralImplants Res 2007;18:73-85.

6. Raigrodski AJ, Hillstead MB, Meng GK, Chung KH. Survival and complications of zirconia-based fixed dental prostheses: a systematic review. J Prosthet Dent 2012;107:170-7.

7. Miyazaki T, Nakamura T, Matsumura H, Ban S, Kobayashi T. Current status of zirconia restoration. J Prosthodont Res 2013;57:236-61.from 2

8. Fuzzi M, Tricarico MG, Ferrari Cagidiaco E, Bonadeo G, Sorrentino R, Ferrari M. Nano-leakage and internal adaptation of zirconia and lithium disilicate single crowns with knife edge preparation. J Osseointegr 2017;9:262-74.

9. Zarone F, Ferrari M, Mangano FG, Leone R, Sorrentino R. Digitally oriented materials: Focus on lithium disilicate ceramics. Int J Dent 2016;18:9840594.

10. Liu B, Lu C, Wu Y, Zhang X, Arola D, Zhang D. The effects of adhesive type and thickness on stress distribution in molars restored with all-ceramic crowns. J Prosthodont. 2011;20(1):35-44

11. He LH, Swain M. A novel polymer infiltrated ceramic dental material. Dent Mater 2011;27:527-34

12. Coldea A, Swain MV, Thiel N. Mechanical properties of polymer-infiltrated-ceramic-network materials. Dent Mater 2013; 29:419-26.

13. Coldea A, Swain MV, Thiel N. In-vitro strength degradation of dental ceramics and novel PICN material by sharp indentation. J Mech Behav Biomed Mater 2013;26:34-42.

14. Varga S, Spalj S, Lapter Varga M, Anic Milosevic S, Mestrovic S, Slaj M. Maximum voluntary molar bite force in subjects with normal occlusion. Eur J Orthod 2011; $33: 427-33$.

15. Yassini E, Mirzaei M,Alimi A, Rahaeifard M. Investigation of the fatigue behavior of adhesive bonding of the lithium 
disilicate glass ceramic with three resin cements using rotating fatigue method. $\mathrm{J}$ Mech Behav Biomed Mater 2016;61:62-9.

16. Sieper K, Wille S, Kern M. Fracture strength of lithium disilicate crowns compared to polymer-infiltrated ceramicnetwork and zirconia reinforced lithium silicate crowns. J Mech Behav Biomed Mater 2017;74:342-8.

17. Verena Preis, Sebastian Hahnel, Michael Behr, Martin RosentrittIn vitro performance and fracture resistance of novel CAD/CAM ceramic molar crowns loaded on implants and human teethJ Adv Prosthodont 2018;10:300-7

18. Nawafleh N, Hatamleh M, Elshiyab S and Mack F, Lithium Disilicate Restorations Fatigue Testing Parameters: A Systematic ReviewJournal of Prosthodontics 25 (2016) 116-126).

19. Jung R.E., Pjetursson B.E., Glauser R., Zembic A., Zwahlen M., Lang N.P. A systematic review of the 5-year survival and complication rates of implant-supported single crowns. Clin Oral Implants Res 2008;19: 119-130.

20. Jung R.E.,Zembic A., Pjetursson B.E.,Zwahlen M., Thoma D. Systematic review of the survival rate and the incidence of biological, technical and esthetic complications of single crowns on implants reported in longitudinal studies with a mean follow-up of 5 years. Clin Oral Implants Res 2012; 23(6): 2-21. From 7

21. Zhao K, Pan Y, Guess PC, et al: Influence of veneer application on fracture behavior of lithium-disilicate-based ceramic crowns. Dent Mater 2012;28:653-660.

22. Dogan, Derya Ozdemir ; Gorler, Oguzhan ; Mutaf, Burcu ; Ozcan, Mutlu ; Eyuboglu, Gunes Bulut ; Ulgey, MelihFracture Resistance of Molar Crowns Fabricated with Monolithic All-Ceramic CAD/CAM Materials Cemented on Titanium Abutments: An In Vitro StudyJournal of Prosthodontics, 26(4):309- 314.

23. Thompson VP, Rekow DE. Dental ceramics and the molar crown testing ground. J Appl Oral Sci 2004;12:26-36.

24. Chen C, Trindade FZ, de Jager N, Kleverlaan CJ, Feilzer AJ. The fracture resistance of a CAD/CAM Resin Nano Ceramic (RNC) and a CAD ceramic at different thicknesses. DentMater 2014;30:954-62.

25. Taha, N. A., J. E. Palamara, and H. H. Messer. Fracture strength and fracture patterns of root-filled teeth restored with direct resin composite restorations under static and fatigue loading. Operative dentistry; 39(2): 181-188(2014).

26. Kirsten, Armin, et al. Subcritical crack growth behavior of dispersion oxide ceramics. Journal of Biomedical Materials Research Part B: Applied Biomaterials, 95(1): pp. 202-206 (2010).

27. Bilkhair, Asma. Fatigue Behaviour and Failure Modes of Monolithic CAD/CAM Hybrid-ceramic and All-ceramic Posterior Crown Restorations: Ermüdungsverhalten und Bruchfestikeit Von Monolithischen CAD/CAM HybridKeramik- Materials und Vollkeramischen Restaurationen Im Seitenzahnbereich. Diss. Universität, (2014).

28. Zainab M. Jassim and Manhal A. MajeedComparative Evaluation of the Fracture Strength of Monolithic Crowns Fabricated from Different all-ceramic CAD/CAM Materials (an in vitro study) Biomedical \& Pharmacology Journal, September 2018Vol. 11(3), p. 1689-1697

29. kok et al 3. de Kok P, Kleverlaan CJ, de Jager N, Kuijs R, Feilzer AJ Mechanical performance of implant-supported posterior crowns. J Prosthet Dent ; 2015: 114:59-66.

30. Kim MJ, Oh SH, Kim HJ: Wear evaluation of the human enamel opposing different zirconia dental ceramic and other porcelain.J Dent;2012:40(11): 979-88.

31. Keisuke Nakamura, Akio Harada, Ryoichi Inagaki, Taro Kanno, Yoshimi Niwano, Percy Milleding \& Ulf Örtengreni: Fracture resistance of monolithic zirconia molar crowns with reduced thickness Acta Odontologica Scandinavica. 2015; Early Online, 1-7

32. Beuer F, Stimmelmayr M, Gueth JF, Edelhoff D, Naumann M. In vitro performance of full-contour zirconia single crowns. Dent Mater 2012;28:449-56.

33. John O. Burgess, DDS, MS: Zirconia: The Material, Its Evolution, and Composition. Oct,2018.vol 39, Issue 4. Compendium of continuous education in dentistry

34. Della Bona, Alvaro, Pedro H. Corazza, and Yu Zhang. Characterization of a polymer-infiltrated ceramic-network material. Dental Materials, m 30(5): pp.564-569 (2014).

35. Petrini, Morena, Maurizio Ferrante, and Bo Su. Fabrication and characterization of biomimetic ceramic/ polymer composite materials for dental restoration. Dental Materials, 29(4): pp. 375-381 (2013).

36. Homaei, Ehsan, et al. Static and fatigue mechanical behavior of three dental CAD/CAM ceramics. Journal of the mechanical behavior of biomedical materials, 59: pp. 304-313 (2016).

37. Sieper, Kim, Sebastian Wille, and Matthias Kern. "Fracture strength of lithium disilicate crowns compared to polymerinfiltrated ceramicnetwork and zirconia reinforced lithium 
silicate crowns." Journal of the mechanical behavior of Biomedical materials, 74: pp. 342-348 (2017).

38. Jaiane Bandoli Monteiroa, Hilton Riquieria, Catina Prochnowb, Luís Felipe Guilardib, Gabriel Kalil Rocha Pereirab, c, Alexandre Luiz Souto Borgesd, Renata Marques de Melod, Luiz Felipe Valandrob Fatigue failure load of two resin- ondedzirconia-reinforced lithium silicate glass-ceramics:Effect of ceramic thickness dental materials. $2018 ; 34: 891-900$.

39. Chen C, Trindade FZ, de Jager N, Kleverlaan CJ, Feilzer AJ. The fracture resistance of a CAD/CAM Resin Nano Ceramic (RNC) and a CAD ceramic at different thicknesses. Dent Mater 2014;30:954-62.

40. Preis V, Behr M, Hahnel S, Rosentritt M. Influence of cementation on in vitro performance, marginal adaptation and fracture resistance of CAD/CAM-fabricated ZLS molar crowns. Dent Mater 2015; 31:1363-9.

41. Skalak R. Biomechanical considerations in osseointegrated prostheses. J Prosthet Dent 1983;49:843-848.

42. Branemark PI.Osseintegration and its experimental background.J Prosthet Dent 1983;50:399-410.

43. Menini M, Conserva E, Tealdo T, Bevilacqua M, Pera F, Signori A, Pera P. Shock absorption capacity of restorative materials for dental implant prostheses: an in vitro study. Int J Prosthodont. 2013;26(6):549-56.

44. Rosentritt M, Hahnel S, Engelhardt F, Behr M, Preis V. In vitro performance and fracture resistance of $\mathrm{CAD} /$ CAMfabricated implant supported molar crowns. Clin Oral Investig. 2016 DOI:10.1007/s00784-016-1898-9

45. Weyhrauch M, Igiel Ch, Scheller H, Weibrich G, Lehmann K M. Fracture strength of monolithic all-ceramic crowns on titanium implant abutments. Int J Oral Maxillofac Implants 2016; 31( 2): 304-09.

46. R. Sorrentino, Y. Na gasa wa, M. Infelis e, G. Bonad eo, M. Ferrari In vitro analysis of the fracture resistance of CAD-CAM monolithic lithium disilicate molar crowns with different occlusal thickness June 2018; 10(2)Jof osseointegration

47. Sieper K, Wille S, Kern M. Fracture strength of lithium disilicate crowns compared to polymer-infiltrated ceramicnetwork and zirconia reinforced lithium silicate crowns. J Mech Behav Biomed Mater 2017;74:342-8.

48. Seydler B, Rues S, Muller D, Schmitter M. In vitro fracture load of monolithic lithium disilicate ceramic molar crowns with different wall thicknesses. Clin Oral Investig 2014; 18:1165-71.
49. Jaiane Bandoli Monteiroa, Hilton Riquieria, Catina Prochnowb, Luís Felipe Guilardib, Gabriel Kalil Rocha Pereirab, c, Alexandre Luiz Souto Borgesd, Renata Marques de Melod, Luiz Felipe Valandrob Fatigue failure load of two resin- ondedzirconia-reinforced lithium silicate glass-ceramics: Effect of ceramic thickness dental materials 34 (2018) 891-900

50. Choi S., Hyung-In Yoon, and Eun-Jin Park Load-bearing capacity of various CAD/CAM monolithic molar crowns under recommended occlusal thickness and reduced occlusal thickness conditions J Adv Prosthodont. 2017 Dec;9(6):423-431.

51. Preis, Verena, et al. Influence of cementation on in vitro performance, marginal adaptation and fracture resistance of CAD/CAM-fabricated ZLS molar crowns. Dental Materials, 31(11): pp.1363-1369 (2015).

52. Schwindling, Franz Sebastian 29 from 15). Schwindling, Franz Sebastian, Stefan Rues, and Marc Schmitter. "Fracture resistance of glazed, full-contour ZLS incisor crowns." Journal of prosthodontic research, 61(3): pp. 344-349 (2017).

53. Dentsply Sirona. Celtra Duo. Technical product information (2017).

54. Manicone, Paolo Francesco, Pierfrancesco Rossi Iommetti, and Luca Raffaelli. An overview of zirconia ceramics: basic properties and clinical applications. Journal of dentistry, 35(11): pp.819-826 (2007).

55. Sieper, Kim, Sebastian Wille, and Matthias Kern. "Fracture strength of lithium disilicate crowns compared to polymerinfiltrated ceramic network and zirconia reinforced lithium silicate crowns." Journal of the mechanical behavior of biomedical materials, 74: pp. 342-348. (2017).

56. Güngör, Merve Bankoðlu, and Secil Karakoca Nemli. "Fracture resistance of CAD-CAM monolithic ceramic and veneered zirconia molar crowns after aging in a mastication simulator." Journal of Prosthetic Dentistry, 119 (3): pp. 473-480 (2018).

57. Kikuchi M, Korioth TW, Hannam AG. The association among occlusal contacts, clenching effort, and bite force distribution in man. J Dent Res 1997; 76:1316-25.

58. Varga S, Spalj S, Lapter Varga M, Anic Milosevic S, Mestrovic S, Slaj M. Maximum voluntary molar bite force in subjects with normal occlusion. Eur J Orthod 2011; 33:427-33. 